\title{
BIODIVERSITY IN WILD VEGETABLES OF KONKAN REGION -
}

\section{MAHARASHTRA}

\author{
Tabassum M. A. Khan ${ }^{1}$ and Umesh B. Kakde ${ }^{2}$ \\ 1 Govt Of Ms"s Ismail Yusuf College, Jogeshwari (E), Mumbai-400060 \\ India 2 Govt. Of Ms"s Ismail Yusuf College, Jogeshwari (E), Mumbai \\ Corresponding Author Email : Tabassum.64@Rediffmail.Com
}

\begin{abstract}
:
The consumption of wild vegetables and plants is one of the strategies, adopted by the local people for sustenance is intrinsically linked to their strong traditional and cultural system and is inseparable. The indigenous communities include wild edibles to their daily food intake and sales from the surplus add to their income. Diversity of plant foods consumed provides nutritional diversity and also food during famine or scarcity of favored foods. It is essential that the locally available these edible herbs and plant parts are rich source of protein, iron, calories and they are also recognized for their characteristic color, flavor and therapeutic value, be used in the diets to prevent the nutrient deficiency and degenerative diseases. The wild vegetable also has medicinal value and hence beside food supplement they also act as nutritional supplement to the people of this area. The article deals with the diversity in wild vegetables and nutritional value consumed by people of Konkan region of Maharashtra. Wild vegetables refer to the species which are not cultivated at large scale commercially. They are grown on waste land by tribal communities or collected from their natural habitat and used as source of food. Ethanobotanical study was carried out by survey of local market, unstructured interview of local villagers and household food survey. Total 58 species belongs 55 genera and 27 families of Wild vegetables were documented. Out of 58 species 24 were herbaceous 22 climbers/prostate creepers/twiner, 3 shrubs and 9 trees. Out of these recorded species some are good source of protein some are carbohydrate and some are variable minerals.
\end{abstract}

Keywords:

Wild vegetables; Diversity; Nutritional diversity; Konkan region; Ethanobotanical

\section{Introduction:}

The diversity in the wild vegetable not only gives variation in diet but also provides nutritional diversity. It contributes to the house hold food security in this region. Wild vegetable refers to the species which are not cultivated at large scale commercially. They are grown on waste land by tribal communities 
or collected from their natural habitat, fields etc. and used as source of food and income. Various studies have found that wild edible species are potential source of nutrition while in many cases they are more nutritious then conventionally eaten crops. Developing countries like India where food insecurity, malnourishment, poverty is more acute, potential of Wild vegetable in providing food, nutrition, source of income and livelihood in rural settings can be acknowledged. Survey and documentation of wild edible plants and their utilization for food have been conducted in several parts of the country. In Maharashtra several studies have been conducted on traditionally used medicinal plants (especially on Western Ghat). There were no work that records the diversity and usability of wild vegetables in Konkan. Therefore present study was planned to document the diversity in wild vegetables used by rural as well as urban people of Konkan.

\section{Material and Method:}

Study Area: - Geographically Konkan is the coastal belt of western state of Maharashtra. It"s a narrow strip, situated in between the Western Ghat and Arabian Sea. The world famous Western Ghats is lying in the Konkan. It rises from Sea level to 300 meter height. Konkan area ranges from 27 to $48 \mathrm{~km}$ in breadth and about $800 \mathrm{~km}$ in length, from Goa to Tapi basin. It has 6 administrative divisions i.e. Mumbai, Mumbai suburb, Thane, Raigad, Ratnagiri and Sindhudurg constituting area of $30,746 \mathrm{~km}^{2}$. The Konkan Coast is known for its rich vegetation, delicious food, palm and coconut trees. Konkan region formed of mostly secondary lateritic plateaus, hilly tracks and the coastal sandy low land belts. Littoral or mangrove forests, open scrub forest, moist deciduous forest and herbaceous flora of laterite plateaus are main components of vegetation. The long Konkan coast line and vantage ports and its proximity to the Arabian coast were well exploited by the earliest travellers. 
Data Collection \& Anlysis: Study was carried out during the year March 2013 to March 2014.The information related to wild vegetable obtained through household food survey, local market survey (i.e. Mumbai, Mumbai suburb, Thane, Raigad), information collected from local villager during trips to various places of Konkan, informal discussion with people of Ratnagiri and Sindhudurg area. Plant specimens identified during the field visits were cross checked against different informants to validate the information. The collected plants and data entries were registered. Species identification was confirmed by Sutaria (1998), Cooke flora (1967), Singh et al., (2001). Medicinal value was noted down from the book "the herbs of Ayurveda" by Ashok Mithaliya et al. (2005).

\section{Result and Discussion:}

Wild Edible Plant Diversity- During the field survey 58 species of wild vegetables were documented that belongs to 55 genera 27 families. Out of these wild vegetables 7 species belongs to the family Amaranthaceae, 6 Species from Cucurbitaceae, 4 Species from Caesalpiniaceae, 3 species each from Apiaceae, Araceae, Chenopodiaceae, Dioscoreaceae, Fabaceae, and Liliaceae. 2 species each from Apocynaceae, Asteraceae, Convolvulaceae, Mimosae, and

Polygonaceae. One species each from Aizoaceae, Asclepiadaceae, Basellaceae, Commelinaceae, Euphorbiaceae, Poaceae, Moringaceae, Nyctaginaceae Nymphaeaceae, Oxalidaceae, Portulacaceae, Rutaceae, and Solanaceae (Table 2). Life forms indicated that herbs were dominating (41\%) followed by climbers $(40 \%)$, trees (15\%) and shrubs (4\%) (Fig. 1). Plant parts and composition: - Out of these wild vegetables $47 \%$ species was used as leafy vegetables, $22 \%$ species stem, $8 \%$ species inflorescence and flower, $28 \%$ species fruit and $5 \%$ species as a whole plant. The species like Amaranthus panniculata, A. polygamous, A. sowa, A. indica, A. campanulata and Coccinia grandis are used extensively whereas Species like Acheranthus aspera, Coelocia argentia, Commelina benghalensis, Mimosa pudica, etc. are used during festival of "Gauri \& 
Ganpati" as a food offering to the Goddess. Plants nutritional value:-Out of these recorded species some are good source of protein some are carbohydrate and some are variable minerals. Species like Amaranthus viridis, Chenopodium album, Centella asiatica, Commelina benghalensis, Moringa oleifra have been found to be very good source of protein (Kulkarni et al., 2003). Tuber of Dioscoria bulbifera leaves of Oxalis corniculata and Cassia tora are good source of carbohydrate, Proteins and dietary fibres. Species of Portulaca oleracea, Centella asiatica and Cassia tora are good source of iron (Kanchan LataVishwakarma et al., 2011).

\section{Conclusion:}

Tourism, developmental projects, modernisation and deforestation depleting these wild species which are inexpensive lucrative source of vitamins, fibres, minerals and other nutrients for many economically deprived natives. Therefore these wild vegetables should be incorporated under managed cultivation or homestead agroforestry system. Introduction of suitable agro-techniques for commercial production of these wild plants should be carried out. Genetic resources of wild vegetables should be conserved for future use to overcome malnutrition in vegetarian diet, food security and for crop improvement of cultivated relatives of these wild vegetables (Kala, 2007). An emphasis on the sustainable harvesting of wild edible plants will help to enhance and maintain

the region"s biodiversity (Angami et al., 2006).

\section{Acknowledgement:}

Authors are grateful to the informants (Mr A. B. Ghadigaonkar, Ms. Sawant, \& Ms. N. Paulkar) for sharing their knowledge and helping in data collection. We are also thankful to Dr. R.G. Atram, Principal for his encouragement. 


\section{Reference:}

Ashok Sheth (2005). “The Herbs of Ayurveda” Ashok Sheth, Editor, Publisher \& Distributor, Vol I- IV.

Angami, A. P. R. Gajurel, R. Rethy, B. Singh and S. K. Kalita (2006). Status and potential of wild edible plants of Arunachal Pradesh. Indian J. Trad. Knowledge 5:541-550.

Cooke T. (1967). The Flora of the Presidency of Bombay. Vol. I, II, III. Botanical Survey of India. Culcutta.

Dipankar Deb \& Abhijit Sarkar (2013). Wild Edible Plants and their utilization in traditional recipes of Tripura, North India. Advances in Biological Research 7(5), 203-211.

Kala, C. P. (2007). Prioritization of cultivated and wild vegetables by the local people in the Uttaranchal hills of Indian Himalaya. Indian J. Trad.

Knowledge 6:239-243.

Kanchan Lata Vishwakarma \& Veenapani Dubey (2011). Nutritional analysis of indigenous wild edible herbs used in eastern Chhattisgarh, India. Emir. J. Food Agric. 23 (6): 554-560.

Rasingam, L. (2012). Ethnobotanical studies on wild edible plants of Irula Tribes of Pillur valley Coimbatore district Tamil Nadu India. Asian Pasific Jr. of T. Biomedicine, S1493 - S1497.

Rajeswar Pegu \& Jitu Gogoi (2013). Ethno botanical study of Wild Edible plants in Poba reserved forest, Assam, India: multiple functions and implications for conservation. Res. J. Agriculture and forestry Sci. Vol. 1(3), 1-10.

Sheetal Gupta (2005). Analysis of nutrient and anti-nutrient content of underutilized green leafy vegetables “. LWT 38: 339-345. 
Singh N. P., P. Lakshminarasimhan, S. Karthikeyan and Prasanna P. V. (2001) "Flora of Maharashtra State -Dicotyledones" Vol. II. Botanical Survey of India, Calcutta.

Sutaria, R. N. (1998). A Text-Book of "SYSTEMATIC BOTANY". Publisher Ramniklal P. Kothari. pp 466.

Yesodharan, K. (2007). Wild edible plants traditionally used by the tribes in the Parambikulam wild life sanctuary Kerala, India. Natural Product Radiance Vol. 6(1) - pp 74-80.

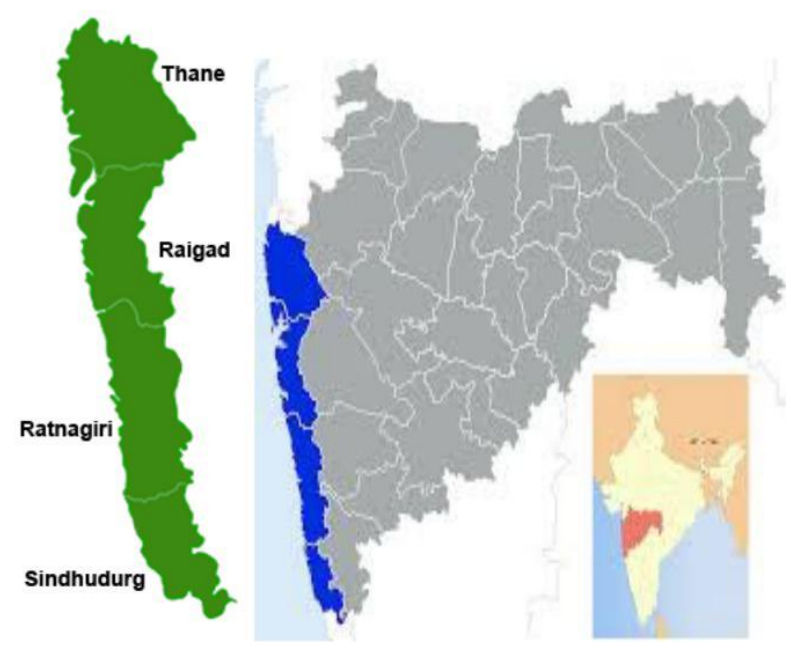

Figure 1:- Map of Konkan showing study area

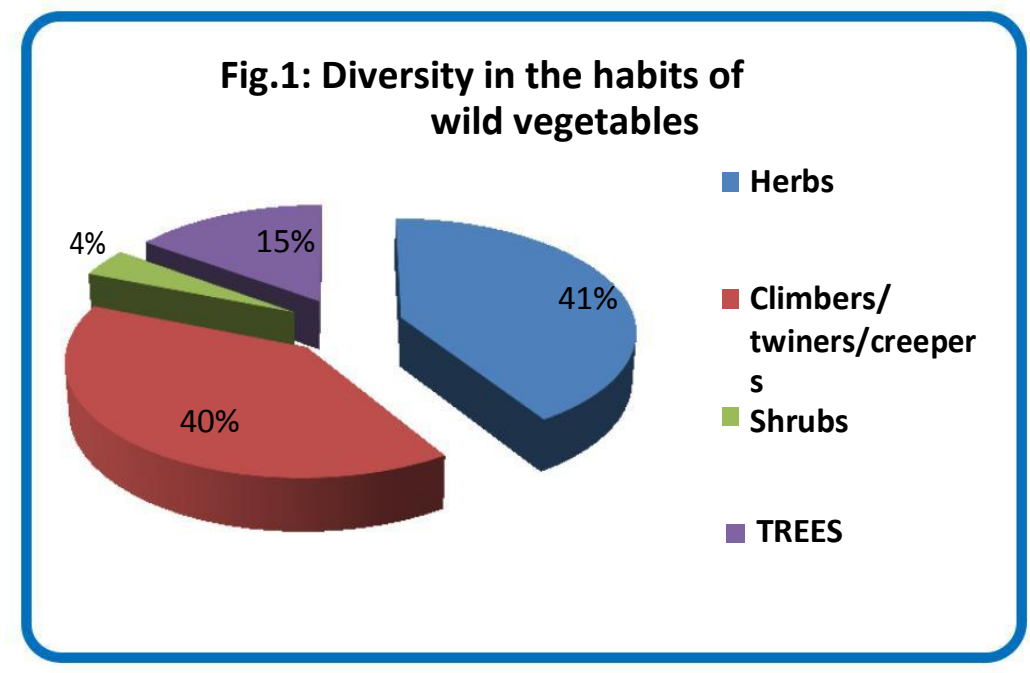


Table 1: List of plants, families and morphological characteristics of wild vegetable of Konkan region

\begin{tabular}{|c|c|c|c|c|c|c|c|}
\hline $\begin{array}{l}\text { Sr. } \\
\text { NO }\end{array}$ & $\begin{array}{l}\text { Scientific \& } \\
\text { common name }\end{array}$ & Family & Morphology & $\begin{array}{l}\text { Part } \\
\text { used }\end{array}$ & $\begin{array}{c}\text { Chemical } \\
\text { constituent }\end{array}$ & Properties & $\begin{array}{l}\text { Medicinal } \\
\text { value }\end{array}$ \\
\hline 1 & $\begin{array}{l}\text { Abrus } \\
\text { precatorius } \\
\text { [Gunja / } \\
\text { Haripatti] }\end{array}$ & Fabaceae & $\begin{array}{c}\text { Deciduous, } \\
\text { wiry climber } \\
\text {, leaves } \\
\text { pinnate many } \\
\text { pair of leaflet }\end{array}$ & $\begin{array}{c}\text { Leaves } \\
\text { to } \\
\text { Prepare } \\
\text { Chatni }\end{array}$ & $\begin{array}{l}\text { Glycyrrhizin, } \\
\text { precol, abrol, } \\
\text { abrasine. }\end{array}$ & $\begin{array}{c}\text { Astringent, emetic, } \\
\text { diuretic and } \\
\text { alexetric. }\end{array}$ & $\begin{array}{l}\text { used in cough, } \\
\text { stomatitis \& } \\
\text { inflammations }\end{array}$ \\
\hline 2 & $\begin{array}{l}\text { Achyranthes } \\
\text { aspera } \\
\text { [Chirchita/ } \\
\text { Aghada] }\end{array}$ & Amaranthaceae & $\begin{array}{c}\text { Annual herb, } \\
\text { quadrangular } \\
\text { stem, simple } \\
\text { opp. leaves }\end{array}$ & $\begin{array}{c}\text { Tender } \\
\text { leaves } \\
\text { to } \\
\text { Prepare } \\
\text { main } \\
\text { dish }\end{array}$ & $\begin{array}{l}\text { Achyranthine } \\
\text { amino acids and } \\
\text { potassium }\end{array}$ & $\begin{array}{l}\text { thermogenic, } \\
\text { expectorant, } \\
\text { carminative, } \\
\text { laxative and } \\
\text { diuretic }\end{array}$ & $\begin{array}{l}\text { used in } \\
\text { asthma, } \\
\text { bronchitis, } \\
\text { flatulence and } \\
\text { colic pain }\end{array}$ \\
\hline 3 & $\begin{array}{l}\text { Alternanthera } \\
\text { sessilis } \\
\text { [Gudrisag] }\end{array}$ & Amaranthaceae & $\begin{array}{l}\text { Branched } \\
\text { prostrate } \\
\text { herb, leaves } \\
\text { simple, }\end{array}$ & $\begin{array}{l}\text { leaves } \\
\text { to } \\
\text { prepare } \\
\text { main } \\
\text { dish }\end{array}$ & $\begin{array}{c}\text { sitosterol, } \\
\text { campesterol, } \\
\text { lupeol and } \\
\text { rhamnoside }\end{array}$ & $\begin{array}{l}\text { astringent, cooling, } \\
\text { digestive and } \\
\text { galactagogue }\end{array}$ & $\begin{array}{l}\text { useful in } \\
\text { diarrhoea, } \\
\text { fever, } \\
\text { amaemia etc. }\end{array}$ \\
\hline 4 & $\begin{array}{l}\text { Amaranthus } \\
\text { paniculatus } \\
\text { [Lalmath, } \\
\text { Rajagira] }\end{array}$ & Amaranthaceae & $\begin{array}{c}\text { Tall annual } \\
\text { herb, leaves } \\
\text { simple ovate } \\
\text { red, }\end{array}$ & $\begin{array}{c}\text { leaves } \\
\text { and } \\
\text { tender } \\
\text { stem } \\
\text { to } \\
\text { prepare } \\
\text { main } \\
\text { dish } \\
\text { parched } \\
\text { grains } \\
\text { to } \\
\text { prepare } \\
\text { chikki }\end{array}$ & $\begin{array}{c}\text { minerals, fibres, } \\
\text { carbohydrates } \\
\text { etc. }\end{array}$ & $\begin{array}{c}\text { diuretic, laxative } \\
\text { haemostatic and } \\
\text { blood purifier }\end{array}$ & $\begin{array}{l}\text { useful in } \\
\text { constipation, } \\
\text { piles and } \\
\text { anaemia }\end{array}$ \\
\hline 5 & $\begin{array}{l}\text { Amaranthus } \\
\text { polygamous } \\
\text { /viridis } \\
\text { [chauli] }\end{array}$ & Amaranthaceae & $\begin{array}{l}\text { Small annual } \\
\text { herb, fleshy } \\
\text { stem, leaves } \\
\text { simple ovate }\end{array}$ & $\begin{array}{l}\text { leaves } \\
\text { and } \\
\text { tender } \\
\text { stem } \\
\text { to } \\
\text { prepare } \\
\text { main } \\
\text { dish }\end{array}$ & $\begin{array}{c}\text { Vitamins, fibres, } \\
\text { carbohydrates, } \\
\text { minerals. Amino } \\
\text { acid reported in } \\
\text { leaves are } \\
\text { arginine,cysteine, } \\
\text { histidine, leucine } \\
\text {, lysine } \\
\text {,tryptophan, } \\
\text { tyrosin. And } \\
\text { valine }\end{array}$ & $\begin{array}{c}\text { astringent, diuretic, } \\
\text { digestive, } \\
\text { appetiser, mild } \\
\text { laxative }\end{array}$ & $\begin{array}{c}\text { useful in } \\
\text { constipation, } \\
\text { piles , } \\
\text { anaemia, } \\
\text { jaundice, } \\
\text { leucorrhoea } \\
\text { etc. }\end{array}$ \\
\hline 6 & $\begin{array}{l}\text { Amaranthus } \\
\text { spinosus } \\
\text { [Katili, kate } \\
\text { math] }\end{array}$ & Amaranthaceae & $\begin{array}{l}\text { Glabrous, } \\
\text { spinous herb, } \\
\text { varying in } \\
\text { colour green } \\
\text { to red, leaves } \\
\text { simple }\end{array}$ & $\begin{array}{c}\text { young } \\
\text { leaves, } \\
\text { stem } \\
\text { to } \\
\text { Prepare } \\
\text { main } \\
\text { dish }\end{array}$ & $\begin{array}{l}\text { Vitamins, fibres, } \\
\text { minerals, Amino } \\
\text { acid, } \beta- \\
\text { sitosterol, } \\
\text { cholesterol etc. }\end{array}$ & $\begin{array}{c}\text { cooling, laxative, } \\
\text { diuretic, } \\
\text { stomachic, } \\
\text { appetiser and tonic }\end{array}$ & $\begin{array}{l}\text { leucorrhoea, } \\
\text { menorrhagia, } \\
\text { anaemia, } \\
\text { anorexia fever } \\
\text { etc. }\end{array}$ \\
\hline
\end{tabular}




\begin{tabular}{|c|c|c|c|c|c|c|c|}
\hline $\begin{array}{l}\mathrm{Sr} . \\
\mathrm{N} \\
\mathrm{O}\end{array}$ & $\begin{array}{l}\text { Scientific \& } \\
\text { common name }\end{array}$ & Family & Morphology & Part used & $\begin{array}{l}\text { Chemical } \\
\text { constituent }\end{array}$ & Properties & $\begin{array}{l}\text { Medicinal } \\
\text { value }\end{array}$ \\
\hline 7 & $\begin{array}{l}\text { Asparagus } \\
\text { racemosus } \\
\text { [shatavari] }\end{array}$ & Liliaceae & $\begin{array}{c}\text { Spinous } \\
\text { branched } \\
\text { climber with } \\
\text { fasciculated } \\
\text { tuberous root } \\
\text { leaves spinous }\end{array}$ & $\begin{array}{l}\text { fasciculate } \\
\mathrm{d} \text { tuberous } \\
\text { root to } \\
\text { prepare } \\
\text { soups }\end{array}$ & $\begin{array}{l}4 \text { types } \\
\text { saponin, } \\
\text { mucilage } \\
\text { starch }\end{array}$ & $\begin{array}{l}\text { Bitter, sweet, } \\
\text { emollient, } \\
\text { astringent, } \\
\text { cooling, } \\
\text { diuretic, } \\
\text { appetiser etc }\end{array}$ & $\begin{array}{c}\text { Useful in } \\
\text { nervous } \\
\text { disorders, } \\
\text { acidity, } \\
\text { dyspepsia \& } \\
\text { Powerful tonic }\end{array}$ \\
\hline 8 & $\begin{array}{l}\text { Alocasia indica } \\
\text { [shewra] }\end{array}$ & Araceae & $\begin{array}{c}\text { Large stout } \\
\text { herb with large } \\
\text { rootstalk. } \\
\text { Leaves large } \\
\text { stagittatecordat } \\
\text { e }\end{array}$ & $\begin{array}{l}\text { Leaves \& } \\
\text { Rhizome } \\
\text { to prepare } \\
\text { main dish }\end{array}$ & $\begin{array}{l}\text { Starch, oxalic } \\
\text { acid, } \\
\text { alocasin- } \\
\text { sterols } \\
\text { minerals }(\mathrm{Ca})\end{array}$ & $\begin{array}{c}\text { Digestive } \\
\text { laxative, } \\
\text { diuretic, } \\
\text { astringent, } \\
\text { nutritive }\end{array}$ & $\begin{array}{c}\text { Useful in } \\
\text { constipation, } \\
\text { pile, swelling, } \\
\text { anorexia ,gout, } \\
\text { rheumatism }\end{array}$ \\
\hline 9 & $\begin{array}{l}\text { Amorphophallu } \\
\text { s } \\
\text { campanulatus } \\
\text { [Suran] }\end{array}$ & Araceae & $\begin{array}{l}\text { Stout herb with } \\
\text { dark brown } \\
\text { corm, leaves } \\
\text { compound }\end{array}$ & $\begin{array}{c}\text { Corm } \\
\text { to prepare } \\
\text { main dish }\end{array}$ & $\begin{array}{c}\text { Protein, } \\
\text { carbohydrate, } \\
\text { calcium, } \\
\text { phosphorus, } \\
\text { vit. A \& B } \\
\text { etc. }\end{array}$ & $\begin{array}{l}\text { astringent, } \\
\text { thermo genic, } \\
\text { appetiser } \\
\text { stomachic, } \\
\text { liver tonic etc. }\end{array}$ & $\begin{array}{l}\text { inflammation, } \\
\text { flatulence, } \\
\text { constipation, } \\
\text { anorexia }\end{array}$ \\
\hline 10 & $\begin{array}{l}\text { Anethum sowa } \\
\text { (graveolens) } \\
\text { [suva/shepu] }\end{array}$ & $\begin{array}{c}\text { Apiaceae } \\
\text { [umbeliferae] }\end{array}$ & $\begin{array}{c}\text { Glabrous } \\
\text { aromatic annual } \\
\text { herb, leaves 2-3 } \\
\text { pinnate }\end{array}$ & $\begin{array}{l}\text { leaves } \\
\text { to prepare } \\
\text { main dish }\end{array}$ & $\begin{array}{c}\text { carvone, } \\
\text { d- limonene, } \\
\text { d- } \\
\text { phellandrene }\end{array}$ & $\begin{array}{c}\text { pungent, } \\
\text { thermogenic, } \\
\text { digestive, } \\
\text { carminative, } \\
\text { anthelmintic, } \\
\text { antispasmodic }\end{array}$ & $\begin{array}{c}\text { inflammation, } \\
\text { flatulence, } \\
\text { intestinal } \\
\text { worms, ulcers, } \\
\text { spermatorrhoea } \\
.\end{array}$ \\
\hline 11 & $\begin{array}{l}\text { Basella alba } \\
\text { [poi /lalbachu,/ } \\
\text { indian Spinach] }\end{array}$ & Basellaceae & $\begin{array}{c}\text { Perennial } \\
\text { succulent } \\
\text { glabrous } \\
\text { twining herb } \\
\text { with red } \\
\text { branches, } \\
\text { leaves simple }\end{array}$ & $\begin{array}{c}\text { leaves } \\
\text { to prepare } \\
\text { main dish }\end{array}$ & $\begin{array}{c}\text { iodine, } \\
\text { fluorine, } \\
\text { carotenoids, } \\
\text { iron, and vit- } \\
\text { K }\end{array}$ & $\begin{array}{c}\text { Cooling, } \\
\text { emollient, } \\
\text { aphrodisiac, } \\
\text { laxative, } \\
\text { appetiser. }\end{array}$ & $\begin{array}{c}\text { useful in } \\
\text { constipation, } \\
\text { flatulence, } \\
\text { anorexia, ulcers } \\
\text { etc. }\end{array}$ \\
\hline 12 & $\begin{array}{l}\text { Bauhinia } \\
\text { variegata } \\
\text { [kachnar] }\end{array}$ & $\begin{array}{c}\text { Caesalpiniacea } \\
\mathrm{e}\end{array}$ & $\begin{array}{l}\text { Deciduous tree, } \\
\text { leaves simple } \\
\text { grooved at apex }\end{array}$ & flower bud & $\begin{array}{c}\text { tannin, } \beta- \\
\text { sitosterol, } \\
\text { lupeol }\end{array}$ & $\begin{array}{c}\text { astringent, } \\
\text { cooling, } \\
\text { constipating, } \\
\text { anti- } \\
\text { inflammatory }\end{array}$ & $\begin{array}{c}\text { Useful in } \\
\text { diarrhoea, } \\
\text { dysentery, } \\
\text { ulcers, goiter. }\end{array}$ \\
\hline 13 & $\begin{array}{l}\text { Boerhavia } \\
\text { diffusa } \\
\text { [patherchatta] }\end{array}$ & Nyctaginaceae & $\begin{array}{c}\text { Perennial } \\
\text { diffuse herb } \\
\text { with many } \\
\text { procumbent } \\
\text { branches, } \\
\text { leaves simple }\end{array}$ & $\begin{array}{c}\text { young } \\
\text { leaves } \\
\text { and tender } \\
\text { stem } \\
\text { to prepare } \\
\text { main dish }\end{array}$ & $\begin{array}{c}\text { alkaloids, } \\
\text { tricontanol, } \\
\beta \text {-sitosterol, } \\
\text { ursolic acid } \\
\& \\
\text { potassium } \\
\text { salts }\end{array}$ & $\begin{array}{c}\text { astringent, } \\
\text { cooling, } \\
\text { anthelmintic, } \\
\text { cardiac } \\
\text { stimulant, } \\
\text { laxative \& } \\
\text { tonic }\end{array}$ & $\begin{array}{c}\text { Useful in } \\
\text { cardiac } \\
\text { disorder, } \\
\text { leucorrhoea, } \\
\text { oedema etc. }\end{array}$ \\
\hline 14 & $\begin{array}{l}\text { Carthemus } \\
\text { tinctorius } \\
\text { [kardi/saflower }\end{array}$ & Compositae & $\begin{array}{c}\text { Small spiny } \\
\text { annual herb } \\
\text { Leaves oblong } \\
\text { lanceolate } \\
\text { spiny narrow at } \\
\text { base }\end{array}$ & $\begin{array}{c}\text { Tender } \\
\text { Leaves \& } \\
\text { flower }\end{array}$ & $\begin{array}{l}\text { Carbohydrate } \\
\text {, minerals, oil }\end{array}$ & $\begin{array}{l}\text { Expectorant, } \\
\text { anti- } \\
\text { inflammatory }\end{array}$ & $\begin{array}{l}\text { beneficial in } \\
\text { cold cough, } \\
\text { bronchitis, liver } \\
\text { tonic }\end{array}$ \\
\hline
\end{tabular}




\begin{tabular}{|c|c|c|c|c|c|c|c|}
\hline $\begin{array}{l}\text { Sr. } \\
\text { N } \\
0\end{array}$ & $\begin{array}{l}\text { Scientific \& } \\
\text { common name }\end{array}$ & Family & $\begin{array}{c}\text { Morpholo } \\
\text { gy }\end{array}$ & $\begin{array}{l}\text { Part } \\
\text { used }\end{array}$ & Chemical constituent & Properties & $\begin{array}{l}\text { Medicinal } \\
\text { value }\end{array}$ \\
\hline 15 & $\begin{array}{l}\text { Canavalia } \\
\text { gladiate } \\
\text { [sword bean] }\end{array}$ & Fabaceae & $\begin{array}{l}\text { Perennial } \\
\text { twining } \\
\text { plant, } \\
\text { leaves } \\
\text { trifoliate. }\end{array}$ & $\begin{array}{c}\text { Unripe } \\
\text { tender } \\
\text { pod } \\
\text { to } \\
\text { prepare } \\
\text { main } \\
\text { dish } \\
\end{array}$ & $\begin{array}{l}\text { canavanine, protein, } \\
\text { carbohydrates, } \\
\text { gibberellins, minerals }\end{array}$ & $\begin{array}{l}\text { astringent, cooling, } \\
\text { appetizer, } \\
\text { digestive }\end{array}$ & $\begin{array}{l}\text { beneficial in } \\
\text { anorexia, } \\
\text { dyspepsia, } \\
\text { hyperdipsia }\end{array}$ \\
\hline 16 & $\begin{array}{l}\text { Cassia tora } \\
\text { [Takala] }\end{array}$ & $\begin{array}{c}\text { Caesalpiniace } \\
\text { ae }\end{array}$ & $\begin{array}{c}\text { Annual } \\
\text { herb, } \\
\text { leaves } \\
\text { pinnately } \\
\text { compound }\end{array}$ & leaves & $\begin{array}{c}\text { anthraglucosides, } \\
\text { chrysophanola, } \\
\text { rhein }\end{array}$ & $\begin{array}{c}\text { thermogenic, } \\
\text { laxative,anthelmint } \\
\text { ic, liver tonic }\end{array}$ & $\begin{array}{c}\text { beneficial in } \\
\text { helminthiasis } \\
\text {, fever, } \\
\text { constipation, } \\
\text { cardiac } \\
\text { disorders } \\
\end{array}$ \\
\hline 17 & $\begin{array}{l}\text { Carissa } \\
\text { carandas } \\
\text { (Karvanda) }\end{array}$ & Apocynaceae & $\begin{array}{l}\text { Climbing } \\
\text { shrub, } \\
\text { stem spiny }\end{array}$ & $\begin{array}{l}\text { Fruits } \\
\text { ripe, } \\
\text { unripe }\end{array}$ & mineral \& Vit-C & sour acrid, cooling & $\begin{array}{l}\text { useful in } \\
\text { anorexia, }\end{array}$ \\
\hline 18 & $\begin{array}{l}\text { Celosia } \\
\text { argentea } \\
{[\text { kurdu }} \\
\text { /safedmurga] }\end{array}$ & $\begin{array}{c}\text { Amaranthacea } \\
\mathrm{e}\end{array}$ & $\begin{array}{l}\text { Annual } \\
\text { herb, } \\
\text { leaves } \\
\text { simple } \\
\text { glabrous }\end{array}$ & $\begin{array}{c}\text { young } \\
\text { leaves } \\
\text { to } \\
\text { prepare } \\
\text { main } \\
\text { dish } \\
\end{array}$ & $\begin{array}{c}\text { carbohydrate, } \\
\text { vitamins, minerals } \\
\text { etc. }\end{array}$ & $\begin{array}{l}\text { Diuretic, cooling, } \\
\text { aphrodisiac, blood } \\
\text { purifier, astringent. }\end{array}$ & $\begin{array}{c}\text { beneficial in } \\
\text { calculi, } \\
\text { diabetes, } \\
\text { spermatorrho } \\
\text { ea }\end{array}$ \\
\hline 19 & $\begin{array}{l}\text { Centella } \\
\text { asiatica } \\
\text { [brahmi] }\end{array}$ & $\begin{array}{c}\text { Apiaceae } \\
/ \\
\text { umbelliferae }\end{array}$ & $\begin{array}{l}\text { Perennial } \\
\text { creeper, } \\
\text { leaves } \\
\text { simple } \\
\text { elongated } \\
\text { petioles }\end{array}$ & $\begin{array}{l}\text { leaves } \\
\text { and } \\
\text { young } \\
\text { stem } \\
\text { to } \\
\text { prepare } \\
\text { main } \\
\text { dish }\end{array}$ & $\begin{array}{c}\text { asiaticoside, } \\
\text { brahmoside, } \\
\text { centelloside, vit-C, } \\
\text { triterpenoidtrisacchari } \\
\text { des }\end{array}$ & $\begin{array}{c}\text { sweet, cooling, } \\
\text { cardiotonic, nerv } \\
\text { tonic, carminatve, } \\
\text { diuretic. }\end{array}$ & $\begin{array}{c}\text { useful in } \\
\text { insomnia, } \\
\text { cardiac } \\
\text { debility, } \\
\text { asthma, } \\
\text { amentia }\end{array}$ \\
\hline 20 & $\begin{array}{l}\text { Chenopodium } \\
\text { album } \\
\text { [bathua] }\end{array}$ & $\begin{array}{c}\text { Chenopodiace } \\
\text { ae }\end{array}$ & $\begin{array}{l}\text { Small } \\
\text { herb, } \\
\text { leaves } \\
\text { simple }\end{array}$ & $\begin{array}{c}\text { young } \\
\text { leaves } \\
\text { to } \\
\text { prepare } \\
\text { main } \\
\text { dish }\end{array}$ & $\begin{array}{l}\text { Various Amino acids, } \\
\text { vitamins, minerals. }\end{array}$ & $\begin{array}{c}\text { digestive, } \\
\text { carminative, } \\
\text { laxative, diuretic }\end{array}$ & $\begin{array}{c}\text { flatulence, } \\
\text { seminal } \\
\text { weakness, } \\
\text { cardiac } \\
\text { disorders }\end{array}$ \\
\hline 21 & $\begin{array}{l}\text { Chlorophytum } \\
\text { tuberosum } \\
\text { [phodshi/kuchel } \\
\text { a] }\end{array}$ & Liliaceae & $\begin{array}{l}\text { Small herb } \\
\text { with } \\
\text { tuberous } \\
\text { root, } \\
\text { leaves } \\
\text { radical } \\
\text { sessile, } \\
\text { recurved } \\
\text { wavy }\end{array}$ & $\begin{array}{l}\text { Tender } \\
\text { leaves } \\
\& \\
\text { tuberou } \\
\text { s root }\end{array}$ & $\begin{array}{c}\text { Carbohydrate, } \\
\text { minerals, fibers, root } \\
\text {-protein \&saponin }\end{array}$ & $\begin{array}{l}\text { Astringent, } \\
\text { diuretic, }\end{array}$ & $\begin{array}{l}\text { Useful in } \\
\text { colic, } \\
\text { Anorexia, } \\
\text { bronchitis etc }\end{array}$ \\
\hline
\end{tabular}




\begin{tabular}{|c|c|c|c|c|c|c|c|}
\hline $\begin{array}{l}\mathrm{Sr} \\
\dot{\mathrm{N}} \\
\mathrm{O}\end{array}$ & $\begin{array}{l}\text { Scientific } \\
\text { \& common } \\
\text { name }\end{array}$ & Family & Morphology & $\begin{array}{l}\text { Part } \\
\text { used }\end{array}$ & $\begin{array}{c}\text { Chemical } \\
\text { constituent }\end{array}$ & Properties & $\begin{array}{l}\text { Medicinal } \\
\text { value }\end{array}$ \\
\hline 22 & $\begin{array}{l}\text { Coccinia } \\
\text { grandis } \\
\text { [kundru } \\
\text { /Jangaliton } \\
\text { dli] }\end{array}$ & $\begin{array}{c}\text { Cucurbitace } \\
\text { ae }\end{array}$ & $\begin{array}{c}\text { perennial } \\
\text { branched } \\
\text { tendril } \\
\text { climber, } \\
\text { leaves simple } \\
\text { angled or } \\
\text { lobed 5 } \\
\text { nerved }\end{array}$ & $\begin{array}{l}\text { unri } \\
\text { pe } \\
\text { fruit } \\
\text { to } \\
\text { prepa } \\
\text { re } \\
\text { main } \\
\text { dish }\end{array}$ & $\begin{array}{l}\text { mucilage, starch, } \\
\text { caffeic acid, gum, } \\
\text { quercitin, } \\
\text { kaempferol, } \beta \text { - } \\
\text { sitosterol }\end{array}$ & $\begin{array}{c}\text { cooling, astringent, depurative, } a \\
\text { ntipyretic, diuretic, } \\
\text { galactagogue }\end{array}$ & $\begin{array}{l}\text { burning } \\
\text { sensation, } \\
\text { fever, } \\
\text { agalactia, } \\
\text { jaundice }\end{array}$ \\
\hline 23 & $\begin{array}{l}\text { Colocasia } \\
\text { esculenta } \\
\text { [alu / } \\
\text { Arum/arvi] }\end{array}$ & Araceae & $\begin{array}{c}\text { Tuberous } \\
\text { perennial, } \\
\text { herbwith } \\
\text { underground } \\
\text { corm. Leaves } \\
\text { simple with } \\
\text { sheathing } \\
\text { leaf base, } \\
\text { long petiole }\end{array}$ & $\begin{array}{l}\text { leave } \\
\text { s and } \\
\text { corm } \\
\text { to } \\
\text { prepa } \\
\text { re } \\
\text { main } \\
\text { dish }\end{array}$ & $\begin{array}{l}\text { starch, mucilage, } \\
\text { calcium oxalate, } \\
\text { ca-phosphorus, vit- } \\
\text { A B \& C fibres }\end{array}$ & $\begin{array}{c}\text { expectorant, astringent, } \\
\text { thermogenic, appetiser, } \\
\text { galactogogue, laxative }\end{array}$ & $\begin{array}{l}\text { haemorrh } \\
\text { age, } \\
\text { otorrhorea } \\
\text {, adenitis, } \\
\text { alopecia, } \\
\text { cough, } \\
\text { anorexia } \\
\text { etc. }\end{array}$ \\
\hline 24 & $\begin{array}{l}\text { Commelin } \\
\boldsymbol{a} \\
\text { benghalens } \\
\text { is } \\
{[\text { kena / }} \\
\text { kanchata] }\end{array}$ & $\begin{array}{c}\text { commelinac } \\
\text { eae }\end{array}$ & $\begin{array}{l}\text { Annual herb, } \\
\text { succulent } \\
\text { stem. Leaves } \\
\text { simple, } \\
\text { ovate- elliptic } \\
\text { sessile }\end{array}$ & $\begin{array}{l}\text { Leav } \\
\text { es } \\
\text { to } \\
\text { prepa } \\
\text { re } \\
\text { main } \\
\text { dish }\end{array}$ & $\begin{array}{c}\text { vitamins, } \\
\text { flavocommelin, } \\
\text { saponin, tannin \& } \\
\text { minerals }\end{array}$ & $\begin{array}{c}\text { diuretic, antiseptic, laxative, } \\
\text { cooling, digestive, demulcent } \\
\text { etc. }\end{array}$ & $\begin{array}{c}\text { beneficial } \\
\text { in piles, } \\
\text { constipati } \\
\text { on, fever } \\
\text { calculi, } \\
\text { indigestio } \\
\text { n etc. }\end{array}$ \\
\hline 25 & $\begin{array}{l}\text { Cressa } \\
\text { cretica } \\
\text { [rudanti/ } \\
\text { kharda] }\end{array}$ & $\begin{array}{c}\text { Convolvula } \\
\text { ceae }\end{array}$ & $\begin{array}{l}\text { small dwarf } \\
\text { herb near } \\
\text { shores, } \\
\text { leaves } \\
\text { simple,numer } \\
\text { ous, } \\
\text { subsessile } \\
\text { ovate densely } \\
\text { silky hairy }\end{array}$ & $\begin{array}{l}\text { whol } \\
\text { e } \\
\text { plant } \\
\text { to } \\
\text { prepa } \\
\text { re } \\
\text { main } \\
\text { dish }\end{array}$ & $\begin{array}{l}\text { scopoletin, umbelif } \\
\text { erone, } \\
\text { isopimpinellin } \beta \text { - } \\
\text { sitosterol }\end{array}$ & $\begin{array}{c}\text { acrid, salty, galactogogue, } \\
\text { blood purifier, thermogenic, } \\
\text { anthelmintic, digestive, } \\
\text { carminative etc. }\end{array}$ & $\begin{array}{c}\text { useful in } \\
\text { whooping } \\
\text { cough, } \\
\text { constipati } \\
\text { on, } \\
\text { diabetes, } \\
\text { agalactia, } \\
\text { flatulence, } \\
\text { colic, } \\
\text { anorexia, } \\
\text { helminthi } \\
\text { asis }\end{array}$ \\
\hline 26 & $\begin{array}{l}\text { Dendrocla } \\
\text { mus } \\
\text { strictus } \\
{[\text { Bamboo] }}\end{array}$ & Gramineae & $\begin{array}{c}\text { Tall dense } \\
\text { strong stem } \\
\text { leaves } \\
\text { petiolate base } \\
\text { rounded } \\
\text { gradually } \\
\text { narrowed } \\
\text { upward } \\
\text { twisted tip }\end{array}$ & $\begin{array}{l}\text { tende } \\
\text { r } \\
\text { shoot }\end{array}$ & $\begin{array}{l}\text { carbohydrate, } \\
\text { fibres, minerals }\end{array}$ & Nutritive, thermogenic & $\begin{array}{l}\text { Useful in } \\
\text { T.B. }\end{array}$ \\
\hline 27 & $\begin{array}{l}\text { Digera } \\
\text { muricata } \\
\text { [manjarik] }\end{array}$ & $\begin{array}{c}\text { Amaranthac } \\
\text { eae }\end{array}$ & $\begin{array}{c}\text { annual herb, } \\
\text { stem } \\
\text { glabrous, } \\
\text { hollow, } \\
\text { leaves ovate } \\
\text { simple } \\
\text { reddish } \\
\text { margins }\end{array}$ & $\begin{array}{l}\text { leave } \\
\text { s \& } \\
\text { stem } \\
\text { to } \\
\text { prepa } \\
\text { re } \\
\text { dish }\end{array}$ & $\begin{array}{l}\text { vitamins, minerals, } \\
\text { fibres etc }\end{array}$ & $\begin{array}{c}\text { laxative, astringent, stomachic, } \\
\text { diuretic, demulcent }\end{array}$ & $\begin{array}{c}\text { useful in } \\
\text { diabetes, } \\
\text { constipati } \\
\text { on, } \\
\text { urinary } \\
\text { disorders, } \\
\text { piles. }\end{array}$ \\
\hline
\end{tabular}




\begin{tabular}{|c|c|c|c|c|c|c|c|}
\hline $\begin{array}{l}\text { Sr. } \\
\mathrm{N} \\
\mathrm{O}\end{array}$ & $\begin{array}{l}\text { Scientific \& } \\
\text { common name }\end{array}$ & Family & Morphology & Part used & $\begin{array}{l}\text { Chemical } \\
\text { constituent }\end{array}$ & Properties & $\begin{array}{l}\text { Medicinal } \\
\text { value }\end{array}$ \\
\hline 28 & $\begin{array}{l}\text { Dioscorea alata } \\
\text { [ China Kand] }\end{array}$ & $\begin{array}{c}\text { Dioscoreace } \\
\text { ae }\end{array}$ & $\begin{array}{c}\text { Climber with 4- } \\
\text { winged stems \& } \\
\text { underground tubers, } \\
\text { leaves simple cordate. }\end{array}$ & $\begin{array}{l}\text { Stem } \\
\text { tubers } \\
\text { to prepare } \\
\text { dish }\end{array}$ & $\begin{array}{l}\text { starch, } \\
\text { protein, } \\
\text { minerals, } \\
\text { sucrose, } \\
\text { maltose }\end{array}$ & $\begin{array}{l}\text { Astringent, } \\
\text { digestive, } \\
\text { cooling, } \\
\text { aphrodisiac, } \\
\text { diuretic, } \\
\text { anthelmintic. }\end{array}$ & $\begin{array}{l}\text { useful in piles, } \\
\text { gonorrhoea, } \\
\text { helminthiasis }\end{array}$ \\
\hline 29 & $\begin{array}{l}\text { Dioscorea } \\
\text { bulbifera } \\
\text { [Jaminkand] }\end{array}$ & $\begin{array}{c}\text { Dioscoreace } \\
\text { ae }\end{array}$ & $\begin{array}{c}\text { Perennial, bulbil } \\
\text { bearing twiner with } \\
\text { tuberous root leaves } \\
\text { broad. Ovate cordate }\end{array}$ & $\begin{array}{l}\text { tuberous } \\
\text { root \& } \\
\text { bulbil } \\
\text { to prepare } \\
\text { dish }\end{array}$ & $\begin{array}{l}\text { glucoside, } \\
\text { proteins, } \\
\text { starch, } \\
\text { minerals } \\
\text { etc. }\end{array}$ & $\begin{array}{c}\text { nutritive, } \\
\text { anthelmintic, } \\
\text { aphrodisiac, } \\
\text { diuretic, } \\
\text { blood } \\
\text { purifier, } \\
\text { astringent }\end{array}$ & $\begin{array}{l}\text { Useful in } \\
\text { syphilis, } \\
\text { gonorrhoea, } \\
\text { hydrocele. } \\
\text { Goiter, piles, } \\
\text { dysentery. }\end{array}$ \\
\hline 30 & $\begin{array}{l}\text { Dioscorea } \\
\text { digitata } \\
{[\text { Jaminkand] }}\end{array}$ & $\begin{array}{c}\text { Dioscoreace } \\
\text { ae }\end{array}$ & $\begin{array}{c}\text { Perennial, bulbil } \\
\text { bearing twiner with } \\
\text { tuberous root leaves } \\
\text { broad. Ovate digitate. }\end{array}$ & $\begin{array}{l}\text { tuberous } \\
\text { root \& } \\
\text { infloresce } \\
\text { nce } \\
\text { to prepare } \\
\text { dish }\end{array}$ & $\begin{array}{l}\text { glucoside, } \\
\text { proteins, } \\
\text { starch, } \\
\text { minerals } \\
\text { etc. }\end{array}$ & $\begin{array}{c}\text { nutritive, } \\
\text { anthelmintic, } \\
\text { aphrodisiac, } \\
\text { diuretic, } \\
\text { blood } \\
\text { purifier, } \\
\text { astringent } \\
\end{array}$ & $\begin{array}{l}\text { Useful in } \\
\text { syphilis, } \\
\text { gonorrhoea, } \\
\text { hydrocele. } \\
\text { Goiter, piles, } \\
\text { dysentery. }\end{array}$ \\
\hline 31 & $\begin{array}{l}\text { Holarrhena } \\
\text { antidysentrica } \\
\text { [Kurchi, kuda] }\end{array}$ & $\begin{array}{c}\text { Apocynacea } \\
\mathrm{e}\end{array}$ & Tree & $\begin{array}{l}\text { Flowers } \\
\text { and pods }\end{array}$ & $\begin{array}{c}\text { Holarrhensi } \\
\text { ne, } \\
\text { Kurchine } \\
\text { alkaloids }\end{array}$ & $\begin{array}{l}\text { Carminative, } \\
\text { astringents, } \\
\text { anthihelment } \\
\text { hic }\end{array}$ & $\begin{array}{c}\text { useful in } \\
\text { diarrhoea,dysen } \\
\text { try. }\end{array}$ \\
\hline 32 & $\begin{array}{l}\text { Ipomoea } \\
\text { aquatica } \\
\text { [kalmisag/ } \\
\text { phopali] }\end{array}$ & $\begin{array}{c}\text { Convolvulac } \\
\text { eae }\end{array}$ & $\begin{array}{c}\text { prostate procumbent } \\
\text { herb, leaves simple } \\
\text { ovate, lanceolate } \\
\text { glabrous }\end{array}$ & $\begin{array}{l}\text { leaves and } \\
\text { young } \\
\text { stem } \\
\text { to prepare } \\
\text { dish }\end{array}$ & $\begin{array}{c}\text { minerals } \\
\text { Ca, P. , Vit- } \\
\text { B,C,E etc. }\end{array}$ & $\begin{array}{l}\text { astringent, } \\
\text { expectorant, } \\
\text { emetic, } \\
\text { alexipharmic } \\
\text { etc. }\end{array}$ & $\begin{array}{c}\text { useful in } \\
\text { bronchitis, } \\
\text { asthma, } \\
\text { nervous } \\
\text { disorders } \\
\text {,fatigue } \\
\text { anaemia etc. }\end{array}$ \\
\hline 33 & $\begin{array}{l}\text { Lagenaria } \\
\text { vulgaris } \\
\text { [ Jangalilauki/ } \\
\text { bottle gourd] }\end{array}$ & $\begin{array}{c}\text { Cucurbitacea } \\
\mathrm{e}\end{array}$ & $\begin{array}{c}\text { Soft pubescent } \\
\text { climbing herb, leaves } \\
\text { simple, cordate } \\
\text { dentate. }\end{array}$ & $\begin{array}{l}\text { whole } \\
\text { plant and } \\
\text { fruit } \\
\text { to prepare } \\
\text { dish }\end{array}$ & $\begin{array}{l}\text { cucurbitaci } \\
\text { ns, } \\
\text { minerals, } \\
\text { carbohydrat } \\
\text { es }\end{array}$ & $\begin{array}{c}\text { Bitter, } \\
\text { Emetic, } \\
\text { purgative, } \\
\text { diuretic, } \\
\text { refrigerant, } \\
\text { astringent. }\end{array}$ & $\begin{array}{c}\text { useful in } \\
\text { constipation, } \\
\text { inflammation, } \\
\text { asthma, fever, } \\
\text { constipation, } \\
\text { jaundice, } \\
\text { calculi etc. }\end{array}$ \\
\hline 34 & $\begin{array}{l}\text { Launaea } \\
\text { procumbens } \\
\text { [bhopatri] }\end{array}$ & Asteraceae & $\begin{array}{c}\text { glabrous, prostrate } \\
\text { herb. Leaves simple } \\
\text { radical cauline oblong } \\
\text { lyrate,narrow }\end{array}$ & $\begin{array}{l}\text { leaves } \\
\text { to prepare } \\
\text { dish }\end{array}$ & $\begin{array}{c}\text { tannin } \\
\text {,minerals } \\
(\mathrm{Ca}, \mathrm{Fe},) \\
\text { Vit- B,A,C } \\
\quad \text { etc. }\end{array}$ & $\begin{array}{c}\text { Sweet, } \\
\text { diuretic, } \\
\text { cholagogue, } \\
\text { astringent, } \\
\text { expectorant, } \\
\text { blood } \\
\text { purifier, } \\
\text { lactagogue. }\end{array}$ & $\begin{array}{l}\text { beneficial in } \\
\text { diarrhoea, } \\
\text { dysentery, } \\
\text { toxaemia, } \\
\text { fever, calculi } \\
\text { etc. }\end{array}$ \\
\hline 35 & $\begin{array}{l}\text { Luffa } \\
\text { acutangulavara } \\
\text { mara } \\
\text { [ Kadawaturi] }\end{array}$ & $\begin{array}{c}\text { Cucurbitacea } \\
\mathrm{e}\end{array}$ & $\begin{array}{l}\text { Largemonoecismtend } \\
\text { rilled climber. Leaves } \\
\text { orbicular,cordate, } \\
\text { palmately 5-7 lobed } \\
\text { scabrid on both sides. }\end{array}$ & $\begin{array}{l}\text { unripe } \\
\text { fruit }\end{array}$ & $\begin{array}{c}\text { amino acids } \\
\text {, oleic \& } \\
\text { linoleic } \\
\text { acid, } \\
\text { carbohydrat } \\
\text { e, } \\
\text { minerals }(\mathrm{Ca} \\
, \mathrm{Fe}, \mathrm{P}), \\
\text { Vit- A\&B }\end{array}$ & $\begin{array}{c}\text { bitter, } \\
\text { astringent, } \\
\text { demulcent, } \\
\text { diuretic , } \\
\text { tonic \& } \\
\text { nutritive }\end{array}$ & $\begin{array}{c}\text { useful in } \\
\text { calculi, } \\
\text { anorexia, piles, } \\
\text { \& constipation }\end{array}$ \\
\hline
\end{tabular}




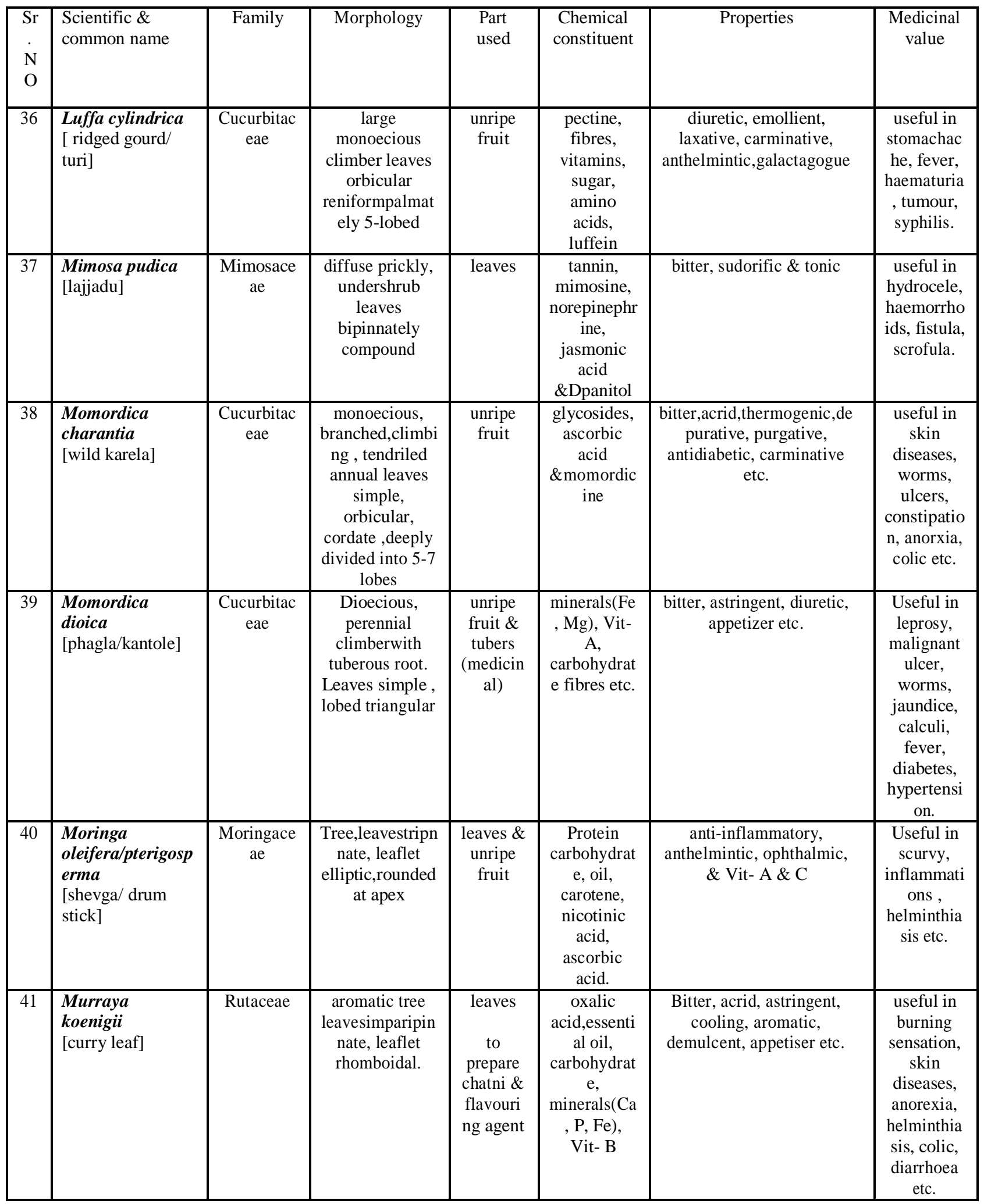




\begin{tabular}{|c|c|c|c|c|c|c|c|}
\hline $\begin{array}{l}\text { Sr. } \\
\text { NO }\end{array}$ & $\begin{array}{l}\text { Scientific \& } \\
\text { common name }\end{array}$ & Family & Morphology & Part used & $\begin{array}{c}\text { Chemical } \\
\text { constituent }\end{array}$ & Properties & $\begin{array}{c}\text { Medicinal } \\
\text { value }\end{array}$ \\
\hline 42 & $\begin{array}{l}\text { Nelumbo } \\
\text { nucifera } \\
\text { [lotus] }\end{array}$ & Nymphaeaceae & $\begin{array}{c}\text { Large } \\
\text { aquatic herb } \\
\text { with } \\
\text { rhizome. } \\
\text { Leaves } \\
\text { simple } \\
\text { peltate, long } \\
\text { petiole }\end{array}$ & $\begin{array}{c}\text { stem } \\
\text { to } \\
\text { prepare } \\
\text { main dish }\end{array}$ & $\begin{array}{l}\text { carbohydrate, } \\
\text { minerals, } \\
\text { fibres, }\end{array}$ & $\begin{array}{l}\text { Astringent, cooling, } \\
\text { fragrant, diuretic. }\end{array}$ & $\begin{array}{c}\text { useful in } \\
\text { vomiting, } \\
\text { leprosy, skin } \\
\text { diseases etc. }\end{array}$ \\
\hline 43 & $\begin{array}{l}\text { Oxalis } \\
\text { corniculata } \\
\text { [tipali/ } \\
\text { tinpatiya] }\end{array}$ & Oxalidaceae & $\begin{array}{c}\text { diffuse } \\
\text { annual or } \\
\text { perennial } \\
\text { creeping } \\
\text { herb, leaves } \\
\text { palmate 3- } \\
\text { foliate.long } \\
\text { stalked }\end{array}$ & $\begin{array}{c}\text { leaves } \\
\text { to } \\
\text { prepare } \\
\text { main dish }\end{array}$ & $\begin{array}{c}\text { glyoxylic } \\
\text { acid, oxalic } \\
\text { acid, vitexin, } \\
\text { glycolipids, } \\
\text { Vit- C, } \\
\text { Phospholipids } \\
\text { etc }\end{array}$ & $\begin{array}{l}\text { sour, astringent, } \\
\text { thermogenic, } \\
\text { cooling, digestive, } \\
\text { carminative, } \\
\text { diuretic, liver tonic }\end{array}$ & $\begin{array}{c}\text { useful in } \\
\text { dyspepsia, } \\
\text { haemorrhoids, } \\
\text { anaemia, fever, } \\
\text { diarrhoea, } \\
\text { dysentery, } \\
\text { scurvy ulcer } \\
\text { etc. }\end{array}$ \\
\hline 44 & $\begin{array}{l}\text { Parkinsonia } \\
\text { aculeate } \\
\text { [ram baval] }\end{array}$ & Caesalpiniaceae & $\begin{array}{l}\text { large armed } \\
\text { shrub with } \\
\text { sharp woody } \\
\text { spines and } \\
\text { prickles. } \\
\text { Leaves } \\
\text { bipinnate } \\
\text { minute } \\
\text { leaflets } \\
\text { flattened } \\
\text { rachis } \\
\end{array}$ & $\begin{array}{l}\text { unripe } \\
\text { green } \\
\text { pods are } \\
\text { eaten }\end{array}$ & $\begin{array}{c}\text { protein, } \\
\text { mucilage, } \\
\text { carbohydrate } \\
\& \text { fatty oil }\end{array}$ & $\begin{array}{l}\text { antipyretic\& anti- } \\
\text { inflammatory }\end{array}$ & $\begin{array}{l}\text { useful in } \\
\text { cough, fever, } \\
\& \text { for quick } \\
\text { energy }\end{array}$ \\
\hline 45 & $\begin{array}{l}\text { Pentatropi } \\
\text { scapensis } \\
\text { [shingroti] }\end{array}$ & asclepiadaceae & $\begin{array}{c}\text { perennial } \\
\text { twiner, } \\
\text { leaves } \\
\text { simple, ovate } \\
\text { acuminate at } \\
\text { apex }\end{array}$ & $\begin{array}{l}\text { leaves } \\
\text { to } \\
\text { prepare } \\
\text { dish }\end{array}$ & $\begin{array}{l}\text { Pentatropin, } \\
\text { minerals etc. }\end{array}$ & $\begin{array}{l}\text { Cooling, astringent, } \\
\text { appetiser, } \\
\text { expectorant. }\end{array}$ & $\begin{array}{c}\text { useful in } \\
\text { cough, } \\
\text { bronchitis, } \\
\text { epilepsy, } \\
\text { anorexia etc }\end{array}$ \\
\hline 46 & $\begin{array}{l}\text { Peucedanum } \\
\text { grande } \\
\text { [wild carrot/ } \\
\text { baphali] }\end{array}$ & Apiaceae & $\begin{array}{c}\text { perennial } \\
\text { erect herb, } \\
\text { leaves } \\
\text { bipinnate } \\
\text {,pinnae } \\
\text { usually 2- } \\
\text { pairs \& } \\
\text { terminal } \\
\text { leaflet, } \\
\text { caulineleaves } \\
\text { 3-lobed } \\
\end{array}$ & $\begin{array}{l}\text { leaves } \\
\text { to } \\
\text { prepare } \\
\text { dish } \\
\text { [monsoon } \\
\text { only] }\end{array}$ & $\begin{array}{l}\text { carbohydrate, } \\
\text { minerals, } \\
\text { fibres, } \\
\text { essential oil }\end{array}$ & $\begin{array}{l}\text { thermogenic, } \\
\text { expectorant, } \\
\text { carminative etc. }\end{array}$ & $\begin{array}{c}\text { useful in } \\
\text { asthma, cough, } \\
\text { bronchitis, } \\
\text { flatulence, } \\
\text { colic } \\
\text { rheumatism, } \\
\text { toothache }\end{array}$ \\
\hline 47 & $\begin{array}{l}\text { Phyllanthus } \\
\text { emblica } \\
\text { [Jangaliaamla] }\end{array}$ & Euphorbiaceae & $\begin{array}{c}\text { small } \\
\text { deciduous } \\
\text { tree, leaves } \\
\text { simple } \\
\text { appears } \\
\text { pinnate }\end{array}$ & $\begin{array}{l}\text { fruit used } \\
\text { making } \\
\text { pickles, } \\
\text { juice, } \\
\text { jam, } \\
\text { chatni }\end{array}$ & $\begin{array}{c}\text { Vit- C, } \\
\text { Minerals }(\mathrm{Ca}, \\
\mathrm{P}, \mathrm{Cu}, \mathrm{Cr},), \\
\text { amino acids, } \\
\text { carbohydrate, } \\
\text { fibres. }\end{array}$ & $\begin{array}{l}\text { astringent, bitter, } \\
\text { acrid, cooling, } \\
\text { ophthalmic, } \\
\text { carminative, } \\
\text { digestive, laxative } \\
\text { etc. }\end{array}$ & $\begin{array}{c}\text { useful in } \\
\text { diabetes, } \\
\text { cough, asthma, } \\
\text { bronchitis, } \\
\text { colic, peptic } \\
\text { ulcer, } \\
\text { opthalmopathy, } \\
\text { anaemia.etc. }\end{array}$ \\
\hline 48 & $\begin{array}{l}\text { Physalis } \\
\text { minima } \\
\text { [sun berry] }\end{array}$ & Solanaceae & $\begin{array}{l}\text { annual herb } \\
\text { leaves } \\
\text { simple } \\
\text { ovate lobed }\end{array}$ & $\begin{array}{l}\text { fruits are } \\
\text { eaten } \\
\text { directly }\end{array}$ & $\begin{array}{l}\text { flavonoids, } \\
\text { sterols, Vit- A } \\
\text { \& C, solanine }\end{array}$ & $\begin{array}{c}\text { sour, sweet, } \\
\text { appetiser }\end{array}$ & $\begin{array}{l}\text { gastropathy, } \\
\text { colic, ulcer, } \\
\text { cough, } \\
\text { bronchitis. }\end{array}$ \\
\hline
\end{tabular}




\begin{tabular}{|c|c|c|c|c|c|c|c|}
\hline $\begin{array}{l}\text { Sr. } \\
\text { NO }\end{array}$ & $\begin{array}{l}\text { Scientific \& } \\
\text { common name }\end{array}$ & Family & Morphology & Part used & $\begin{array}{l}\text { Chemical } \\
\text { constituent }\end{array}$ & Properties & $\begin{array}{l}\text { Medicinal } \\
\text { value }\end{array}$ \\
\hline 49 & $\begin{array}{l}\text { Pithecellobium } \\
\text { dulce } \\
\text { [jungle imli] }\end{array}$ & Mimosaceae & $\begin{array}{l}\text { tree, leaves } \\
\text { compound } \\
\text { with one pair } \\
\text { of pinnae, } \\
\text { elliptic }\end{array}$ & $\begin{array}{l}\text { Fruits } \\
\text { arils are } \\
\text { eaten } \\
\text { directly }\end{array}$ & $\begin{array}{c}\text { Vit- C, } \\
\text { Minerals (Ca, } \\
\text { Mg, Fe, p.) }\end{array}$ & $\begin{array}{l}\text { astringent, sweet, } \\
\text { sour, expectorant, } \\
\text { nutritive }\end{array}$ & $\begin{array}{c}\text { Fatigue, cough, } \\
\text { spondylitis, } \\
\text { fracture, } \\
\text { toxaemia, } \\
\text { jaundice, } \\
\text { diabetes. } \\
\end{array}$ \\
\hline 50 & $\begin{array}{l}\text { Polygonum } \\
\text { glabrum } \\
\text { [jungle } \\
\text { chaurai] }\end{array}$ & Polygonaceae & annual herb & $\begin{array}{l}\text { young } \\
\text { leaves }\end{array}$ & $\begin{array}{l}\text { carbohydrate, } \\
\text { fibres, } \\
\text { minerals }\end{array}$ & $\begin{array}{l}\text { astringent, diuretic, } \\
\text { digestive, mild } \\
\text { laxative }\end{array}$ & $\begin{array}{c}\text { useful in } \\
\text { constipation, } \\
\text { piles, anaemia, } \\
\text { etc. }\end{array}$ \\
\hline 51 & $\begin{array}{l}\text { Portulaca } \\
\text { oleracea } \\
{[\mathrm{ghol}]}\end{array}$ & Portulacaceae & $\begin{array}{c}\text { succulent } \\
\text { prostrate } \\
\text { annual herb } \\
\text { with green } \\
\text { /purple stem } \\
\text { leaves } \\
\text { simple fleshy } \\
\end{array}$ & $\begin{array}{l}\text { whole } \\
\text { plant } \\
\text { to } \\
\text { prepare } \\
\text { main dish }\end{array}$ & $\begin{array}{l}\text { protein, Vit- } \\
\text { A \& B } \\
\text { mucilage, } \\
\text { minerals( } \mathrm{Na}, \\
\mathrm{K}, \mathrm{Mg} \text { ) oxalic } \\
\text { acids }\end{array}$ & $\begin{array}{l}\text { laxative, emollient, } \\
\text { cooling, stomachic, } \\
\text { diuretic etc. }\end{array}$ & $\begin{array}{l}\text { useful in } \\
\text { gastropathy, } \\
\text { anorexia, } \\
\text { constipation, } \\
\text { jaundice, } \\
\text { scurvy etc. }\end{array}$ \\
\hline 52 & $\begin{array}{l}\text { Rumex } \\
\text { elongates } \\
\text { [chukka bhaji] }\end{array}$ & Polygonaceae & $\begin{array}{c}\text { Perennial } \\
\text { erect } \\
\text { glabrous } \\
\text { herb. } \\
\text { leaves } \\
\text { lanceolate, } \\
\text { wavy-cueled } \\
\text { margins }\end{array}$ & $\begin{array}{l}\text { leaves \& } \\
\text { tender } \\
\text { shoot to } \\
\text { prepare } \\
\text { dish }\end{array}$ & $\begin{array}{l}\text { Protein, } \\
\text { carbohydrate, } \\
\text { tannin, } \\
\text { lapthin, } \\
\text { chrysophanol, } \\
\text { Ca-oxalate, } \\
\text { essential oil. }\end{array}$ & $\begin{array}{l}\text { Sour, nutritive, } \\
\text { digestive, diuretic } \\
\text { cooling, blood } \\
\text { purifier. }\end{array}$ & $\begin{array}{l}\text { beneficial in } \\
\text { anaemia, } \\
\text { constipation, } \\
\text { cardiac } \\
\text { problem, } \\
\text { scurvy, } \\
\text { syphilis, piles, } \\
\text { anorexia, colic } \\
\text { etc. }\end{array}$ \\
\hline 53 & $\begin{array}{l}\text { Salicornia } \\
\text { hebacea } \\
\text { [Jointed } \\
\text { glasswort, } \\
\text { soda] } \\
\end{array}$ & Chenopodicaeae & $\begin{array}{l}\text { Herb, leaves } \\
\text { absent, stem } \\
\text { fleshy, } \\
\text { jointed, }\end{array}$ & stem & $\begin{array}{l}\text { starch, } \\
\text { minerals, } \\
\text { fibres }\end{array}$ & laxative, nutritive & $\begin{array}{l}\text { useful in } \\
\text { digestion }\end{array}$ \\
\hline 54 & $\begin{array}{l}\text { Sesbania } \\
\text { grandiflora } \\
\text { [Hatga/agasti] }\end{array}$ & Fabaceae & Tree, & $\begin{array}{l}\text { Flowers } \\
\text { and } \\
\text { tender } \\
\text { fruits }\end{array}$ & $\begin{array}{l}\text { protein, } \\
\text { minerals }\end{array}$ & astringent, nutritive & $\begin{array}{c}\text { useful in } \\
\text { digestion \& } \\
\text { weakness }\end{array}$ \\
\hline 55 & $\begin{array}{l}\text { Smilex indica } \\
\text { (Ghotwel) }\end{array}$ & Liliaceae & climber & $\begin{array}{l}\text { tender } \\
\text { tips }\end{array}$ & $\begin{array}{l}\text { Carbohydrate, } \\
\text { minerals, } \\
\text { fibres }\end{array}$ & $\begin{array}{l}\text { Nutritive, } \\
\text { thermogenic }\end{array}$ & $\begin{array}{l}\text { Useful in } \\
\text { digestion }\end{array}$ \\
\hline 56 & $\begin{array}{l}\text { Suaeda } \\
\text { fruticosa } \\
\text { [Saloonakbuti] }\end{array}$ & Chenopodicaeae & $\begin{array}{l}\text { Branched, } \\
\text { under shrub } \\
\text { Saline soil, } \\
\text { Leaves } \\
\text { Succulent, }\end{array}$ & Leaves & $\begin{array}{l}\text { Minerals } \\
\text { vitamins, } \\
\text { fibres etc. }\end{array}$ & diuretic, laxative & $\begin{array}{l}\text { Useful in } \\
\text { asthma, } \\
\text { rheumatism. }\end{array}$ \\
\hline 57 & $\begin{array}{l}\text { Tamarindus } \\
\text { indica } \\
{[\text { imli] }}\end{array}$ & Caesalpiniaceae & $\begin{array}{l}\text { large tree } \\
\text { leaves } \\
\text { peripinnate }\end{array}$ & $\begin{array}{c}\text { tender } \\
\text { leaves } \\
\text { and fruit } \\
\text { to } \\
\text { prepare } \\
\text { chatni } \\
\end{array}$ & $\begin{array}{c}\text { flavonoid } \\
\text { glycosides, } \\
\text { citric, mallic, } \\
\text {, , tartaric \& } \\
\text { oxalic acids. } \\
\text { Carbohydrate. }\end{array}$ & $\begin{array}{c}\text { sour, astringent, } \\
\text { thermogenic, } \\
\text { anthelmintic, } \\
\text { antifungal, diuretic, } \\
\text { digestive, } \\
\text { carminative. }\end{array}$ & $\begin{array}{c}\text { useful in } \\
\text { gastropathy, } \\
\text { helminthiasis, } \\
\text { ulcer, jaundice, } \\
\text { anorexia, } \\
\text { scurvy. }\end{array}$ \\
\hline 58 & $\begin{array}{l}\text { Trianthema } \\
\text { monogyna } \\
\text { [Shveta] }\end{array}$ & Aizoaceae & $\begin{array}{l}\text { Prostrate, } \\
\text { glabrous, } \\
\text { succulent } \\
\text { annual herb. } \\
\text { Leaves } \\
\text { simple, } \\
\text { obovate. }\end{array}$ & $\begin{array}{l}\text { leaves } \\
\text { to } \\
\text { prepare } \\
\text { dish }\end{array}$ & $\begin{array}{l}\text { minerals( K, } \\
\text { Fe ) } \\
\text { vitamins, } \\
\text { fibres etc. }\end{array}$ & $\begin{array}{c}\text { diuretic, vermifuge, } \\
\text { laxative }\end{array}$ & $\begin{array}{c}\text { useful in } \\
\text { asthma, } \\
\text { amenorrhoea, } \\
\text { oedema, } \\
\text { worms, } \\
\text { rheumatism. }\end{array}$ \\
\hline
\end{tabular}


Table-2: Analytical data of each family

\begin{tabular}{|c|c|c|c|c|c|c|}
\hline Sr.No & families & Total sp. & Trees & Shrubs & Herbs & $\begin{array}{c}\text { Climber/ } \\
\text { Twiner/ } \\
\text { Creeper }\end{array}$ \\
\hline 1. & Aizoaceae & 1 & - & - & - & 1 \\
\hline 2. & Amaranthaceae & 7 & - & - & 7 & - \\
\hline 3. & Apiaceae & 3 & - & - & 2 & 1 \\
\hline 4. & Apocynaceae & 2 & 1 & - & - & 1 \\
\hline 5. & Araceae & 3 & - & - & 3 & - \\
\hline 6. & Asclepiadaceae & 1 & - & - & - & 1 \\
\hline 7. & Asteraceae & $1+1$ & - & - & - & 2 \\
\hline 8. & Basellaceae & 1 & - & - & - & 1 \\
\hline 9. & Caesalpiniaceae & 4 & 2 & 1 & 1 & - \\
\hline 10. & Chenopodiaceae & 3 & - & 1 & 2 & - \\
\hline 11. & commelinaceae & 1 & - & - & 1 & - \\
\hline 12. & Convolvulaceae & 2 & - & - & 2 & - \\
\hline 13. & Cucurbitaceae & 6 & - & - & - & 6 \\
\hline 14. & Dioscoreaceae & 3 & - & - & - & 3 \\
\hline 15. & Euphorbiaceae & 1 & 1 & - & - & - \\
\hline 16. & Fabaceae & 3 & 1 & - & - & 2 \\
\hline 17. & Poaceae & 1 & 1 & - & - & - \\
\hline 18. & Liliaceae & 3 & - & - & 1 & 2 \\
\hline 19. & Mimosaceae & 2 & 1 & 1 & - & - \\
\hline 20. & Moringaceae & 1 & 1 & - & - & - \\
\hline 21. & Nyctaginaceae & 1 & - & - & 1 & - \\
\hline 22. & Nymphaeaceae & 1 & - & - & 1 & - \\
\hline 23. & Oxalidaceae & 1 & - & - & - & 1 \\
\hline 24. & Polygonaceae & 2 & - & - & 2 & - \\
\hline 25. & Portulacaceae & 1 & - & - & - & 1 \\
\hline 26. & Rutaceae & 1 & 1 & - & - & - \\
\hline \multirow[t]{2}{*}{27.} & Solanaceae & 1 & - & - & 1 & - \\
\hline & TOTAL SPP & 58 & 9 & 3 & 24 & 22 \\
\hline
\end{tabular}

